\title{
PSMA Images Thresholding for Prostate Cancer Detection
}

\author{
Ali El-Zaart, Ahmed Bouridane, Rachid Sammouda, and Muhammad Atif Tahir
}

\begin{abstract}
Prostate cancer is the second most common cancer in men, with 10000 new cases and 2500 deaths every year. These numbers increase every year due to the ageing of the general populace. Computer-aided detection (CAD) of prostate cancer can perhaps provide a solution. Computer algorithms allow us to combine the enormous amount of images into a much smaller amount of images with high information content. Image segmentation is an important step of CAD system, the accuracy of the CAD system is related directly to the accuracy of the image segmentation. Thresholding techniques are the most used technique in image segmentation and the statistical approaches are wieldy used in image thresholding. The Gamma distribution was used for radar images processing and mammograms images processing, the results were promised. Our contribution in this paper is to use the Gamma distribution for PSMA segmentation. In this paper, we will use Gamma distribution in order to approximate the data in PSMA image by a mixture of gamma distributions. In this paper we used the maximum likelihood estimator in order to approximate the histogram by a mixture of Gamma distributions. Thresholds between classes are then estimated by minimizing the discrimination error between the classes of pixels in PSMA image. The experimental results on PSMA prostate images using this technique showed good thresholding of images.
\end{abstract}

Keywords-Prostate cancer, PSMA images, thresholding, Gamma distribution, maximum likelihood.

\section{Introduction}

Prostate cancer is the second most common cancer in men, with 10000 new cases and 2500 deaths every year [1, $3]$. These numbers increase every year due to the ageing of the general populace. Several researchers are developing image processing methods to detect early prostate cancer. Statistical approaches are widely used in the processing of PSMA images [4,8], Every region or class of pixels in the PSMA image can be seen as probability density function (distribution), consequently PSMA image or its histogram can be seen as a mixture of distributions. Most of the researchers assume that the data in image can be modeled by a mixture of Gaussian distributions $[5,6]$.

Ali El-Zaart

Faculty of Science, Beirut Arab University

Beirut-Lebanon

Ahmed Bouridane and Muhammad Atif Tahir

Faculty of Engineering and Environment, Northumbria University

Newcastle-UK

Rachid Sammouda

College of Computer and Information Sciences, King Saud University, Kingdom of Saudi Arabia

We know that the Gaussian distribution is a symmetric function and the modes in the histogram of the PSMA images are not usually symmetric functions. For that, the use of Gaussian distribution in the processing of PSMA images is not suitable when the mode is not symmetric.

The Gamma distribution is more general than the Gaussian. It used in the segmentation of the radar images and showed promising results in segmentation [2,7]. The Gamma distribution in homogeneous area is known to be:

$$
\begin{aligned}
& f(x, \mu, N)=\frac{2 q}{\mu} \frac{N^{N}}{\Gamma(N)}\left(\frac{q x}{\mu}\right)^{2 N-1} e^{-N(q x / N)^{2}} \text { (1) } \\
& \text { Where } q=\frac{\Gamma(N+0.5)}{\Gamma(N) \sqrt{N}}, \mathrm{x} \text { is the intensity of the pixel, } \mu \text { is the }
\end{aligned}
$$

mean value of the distribution and $\mathrm{N}$ represents the parameter shape of the distribution. If the value of $\mathrm{N}$ is small then the Gamma distribution is skewed to the right (See Figure $1, \mu=50$ and $\mathrm{N}=1$ ) and if $\mathrm{N}$ is high then the Gamma distribution is symmetry and close to the Gaussian distribution (See Figure $1, \mu=50, \mathrm{~N}=12$ ).

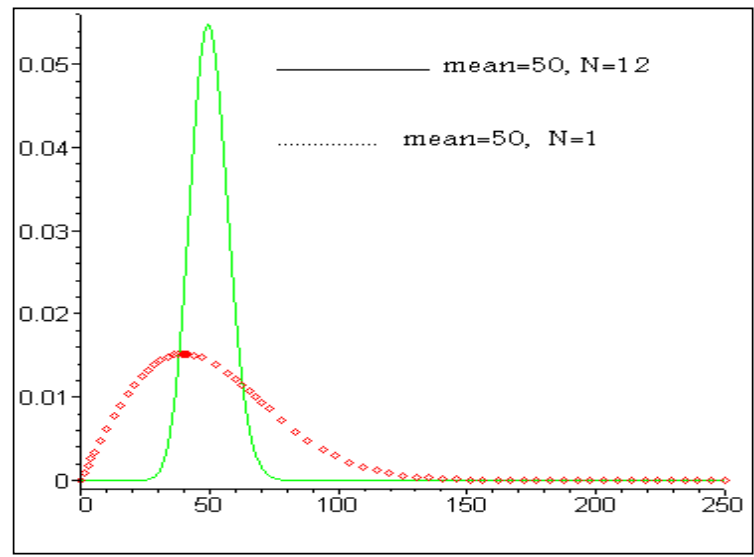

Figure 1: Two Gamma distributions: same values of means and different values of $\mathrm{N}$.

El-Zaart al. [7] used a segmentation method with Gamma distribution to segment the radar images for oil slicks detection. Our contribution in this paper is to adapt this method on the PSMA images for prostate cancer detection. Thresholds are selected at the valleys of a multimodal histogram by minimizing the discrimination error between classes of pixels in PSMA image. In section 2, we will explain the thresholding method. The estimation of thresholds requires an estimation of the statistics (parameters) of PSMA images. The maximum Likelihood Technique with Gamma distribution is therefore used to estimate the histogram parameters in section 3. The experimental result is presented in section 4. Finally, in section 5, we will present a conclusion about the method of segmentation. 


\section{Thresholding of PSMA Images}

We consider a PSMA image $\mathrm{I}(\mathrm{x}, \mathrm{y})$ ) which contains $m$ classes of pixels. A class is defined by a set of homogeneous pixels and each class represents a Gamma distribution, with a specific value of mean $\mu$ and a prior-probability $p$.

Therefore, a PSMA image histogram can be represented by a mixture of Gamma distributions. Thus that, a mode of the histogram can be presented by a Gamma distribution. The thresholded image $s(x, y)$ of $\mathrm{I}(\mathrm{x}, \mathrm{y})$ is given by the following formula:

$$
s(x, y)=\left\{\begin{array}{ccc}
C_{1} & \text { if } & t_{0} \leq I(x, y)<t_{1} \\
C_{2} & \text { if } & t_{1} \leq I(x, y)<t_{2} \\
& & \vdots \\
C_{i} & \text { if } & t_{i-1} \leq I(x, y)<t_{i} \\
\vdots & & \\
C_{k} & \text { if } & t_{k-1} \leq I(x, y)<t_{k}
\end{array}\right.
$$

Where $C_{i}$ is the grey-level value of the class $i$ in the thresholded image, for $i=1 \ldots m$ and $t_{i}$ is the threshold between two modes $i$ and $i+1$, for $i=1 . . m-1$. We estimated the threshold $t_{i}$ using Gamma distribution by minimizing the discrimination error between the classes of pixels in image:

$$
t_{i}=\sqrt{\frac{\log \left(B_{i}\right)}{N q^{2}\left(\frac{1}{\mu_{i}^{2}}-\frac{1}{\mu_{i+1}^{2}}\right)}}
$$

Where $B_{i}=\frac{p_{i}}{p_{i+1}}\left(\frac{\mu_{i+1}}{\mu_{i}}\right)^{2 N} \mu_{i}$ is the mean of the class (mode) $i$ for $\mathrm{i}=1 . . \mathrm{m}$ and $p_{i}$ is the prior-probability of the class $i$ for $i=1 . . m$. In order to estimate the threshold $t_{i}$, we need to estimate the parameters of the histogram i.e., the mean and the prior-probability of each mode. We assume that the parameter shape $N$ of the histogram is constant and it will be given by the user based on the experimentation. In next section, we estimate these parameters.

\section{Histogram Parameters Estimation}

Let $h\left(x_{j}\right)$ be the histogram of PSMA image, where $x_{j}$ $\mathrm{j}=0 . . .255$ is the abscissa of the histogram. The histogram can be seen a mixture of Gamma distributions.

$$
h\left(x_{j}\right)=\sum_{i=1}^{m} I\left(x_{j}, \mu_{i}, N\right) p_{i}
$$

In order to estimate the histogram parameters of the PSMA image, we used the maximum likelihood estimator developed by El-Zaart et al. [2]. The objective of the maximum likelihood estimator is to minimize the error between the real histogram of the PSAM image and the constructed histogram by a mixture of Gamma distributions. The estimated parameters using the maximum likelihood is as follows:

$$
\begin{aligned}
\mu_{i}^{2} & =\frac{\sum_{j=1}^{225} h\left(x_{j}\right) p\left(i / x_{j}, \mu_{i}\right)\left(q x_{k}\right)^{2}}{\sum_{j=1}^{255} h\left(x_{j}\right) p\left(i / x_{j}, \mu_{i}\right)} \\
p_{i} & =\frac{\sum_{k=1}^{H} h\left(x_{j}\right) p\left(i / x_{j}, \mu_{i}\right)}{\sum_{j=1}^{H} h\left(x_{j}\right)}
\end{aligned}
$$

The above equation are iterative and in order to estimate them, we need initial values of means and priorprobabilities. To solve this problem, we used k-mean algorithm in order to estimate the initial parameters of the histogram $\mu_{i}^{0}$ and $p_{i}^{0}$ for $i=1 \ldots m$.

\section{Iv. Experimental Results}

In this section we applied the maximum likelihood estimator on three real PSMA images and then we estimated the thresholds of each images. The number of modes (classes) of these images is $m=3$ and the shape parameter $N=12$. In the following, we present the results of each image:

- Image presented in figure 2 is the PSMA original image, table 1 shows the estimated initial histogram parameters using k-mean, the estimated of final histogram parameters using Maximum likelihood with Gamma distribution, and finally the estimated threshold $t_{1}=39$ and $t_{2}=88$. We applied the estimated thresholds on the original image and we got the thresholded images presented in figure 3. We can remark that the image is well thresholded.

- Image presented in figure 4 is the PSMA original image, table 2 shows the estimated initial histogram parameters using k-mean, the estimated of final histogram parameters using Maximum likelihood with Gamma distribution, and finally the estimated threshold $t_{1}=35$ and $t_{2}=85$. We applied the estimated thresholds on the original image and we got the thresholded images presented in figure 5 . We can remark that the image is well thresholded.

- Image presented in figure 6 is the PSMA original image, table 3 shows the estimated initial histogram parameters using k-mean, the estimated of final histogram parameters using Maximum likelihood with Gamma distribution, and finally the estimated threshold $t_{1}=45$ and $t_{2}=90$. We applied the estimated thresholds on the original image and we got the thresholded images presented in figure 7. We can remark that the image is well thresholded. 


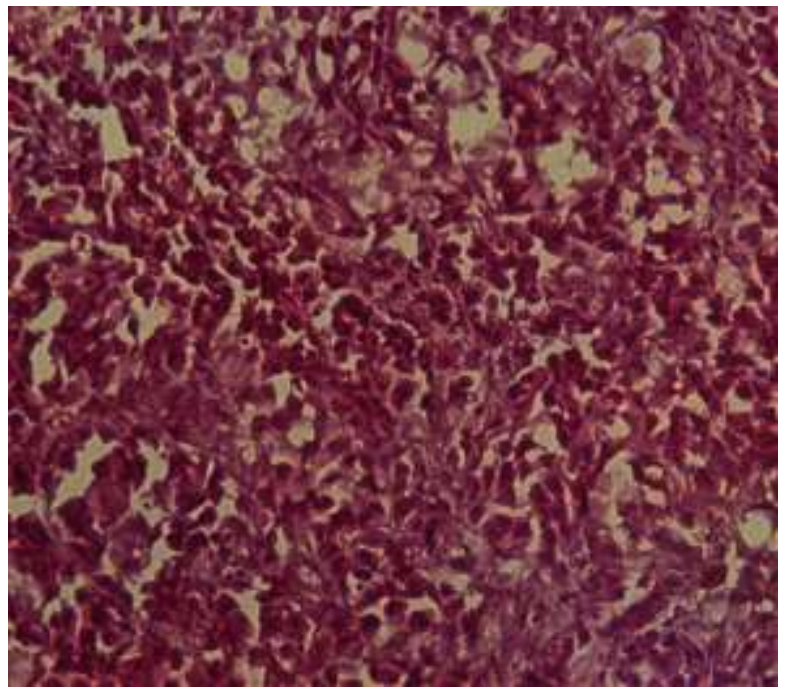

Figure 2: Original PSMA image

TABLE I: ESTIMATION OF INITIAL AND FINAL PARAMETERS, AND ESTIMATION OF THRESHOLDS

\begin{tabular}{|c|c|}
\hline $\begin{array}{c}\text { Initial parameters } \\
\text { Estimated by K-mean }\end{array}$ & $\begin{array}{c}\text { Estimated parameters by } \\
\text { Maximum likelihood }\end{array}$ \\
\hline$\mu_{1}^{0}=32, \quad p_{1}^{0}=0.62$ & $\mu_{1}=27, \quad p_{1}=0.32$ \\
$\mu_{2}^{0}=82, p_{2}^{0}=0.25$ & $\mu_{2}=59, p_{2}=0.39$ \\
$\mu_{3}^{0}=137 p_{3}^{0}=0.11$ & $\mu_{3}=138 p_{3}=0.27$ \\
\hline & Thresholds Estimation \\
\hline & $t_{1}=39$ and $t_{2}=88$ \\
\hline
\end{tabular}

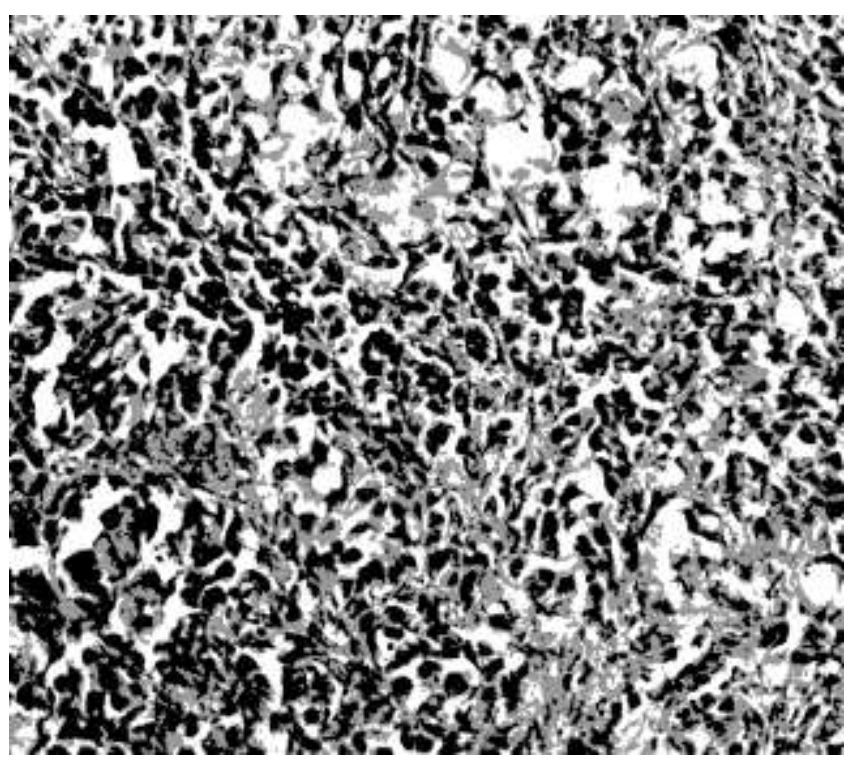

Figure 3: Thresholded images.

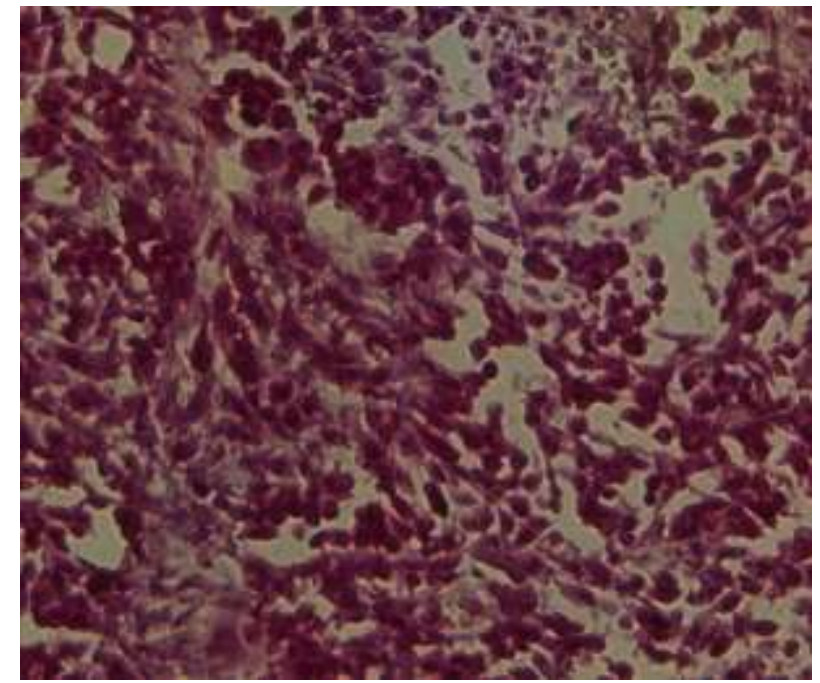

Figure 4: Original PSMA image.

TABLE II: ESTIMATION OF INITIAL AND FINAL PARAMETERS, AND ESTIMATION OF THRESHOLDS

\begin{tabular}{|c|c|}
\hline $\begin{array}{c}\text { Initial parameters } \\
\text { Estimated by K-mean }\end{array}$ & $\begin{array}{c}\text { Estimated parameters by } \\
\text { maximum likelihood }\end{array}$ \\
\hline$\mu_{1}^{0}=31, \quad p_{1}^{0}=0.54$ & $\mu_{1}=24, \quad p_{1}=0.27$ \\
$\mu_{2}^{0}=72, p_{2}^{0}=0.27$ & $\mu_{2}=57, p_{2}=0.34$ \\
$\mu_{3}^{0}=120, p_{3}^{0}=0.18$ & $\mu_{3}=140 p_{3}=0.37$ \\
\hline & Thresholds Estimation \\
\hline & $t_{1}=35$ and $t_{2}=85$ \\
\hline
\end{tabular}

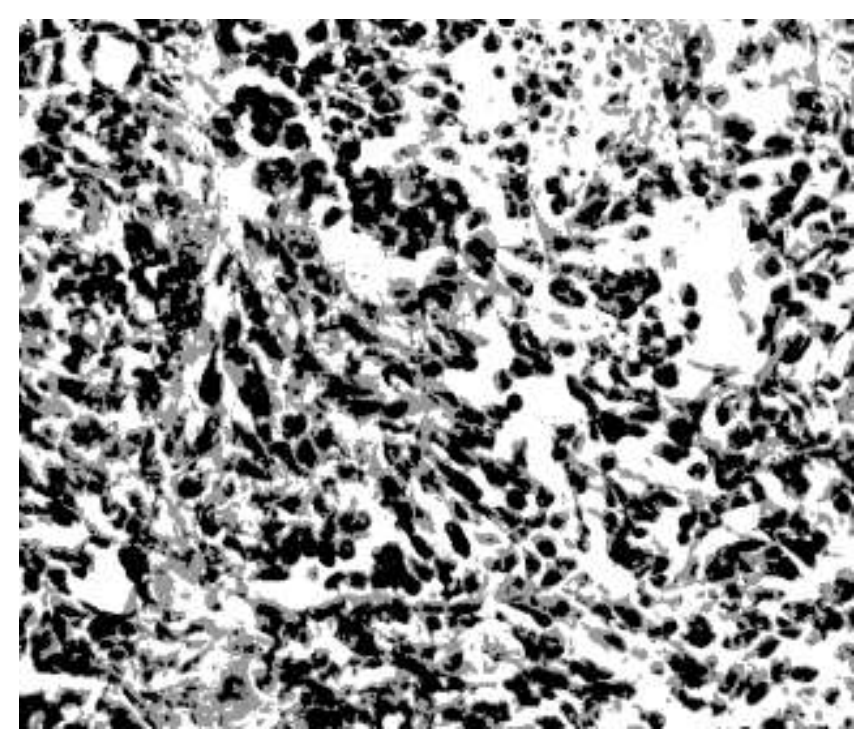

Figure 5: Thresholded images. 


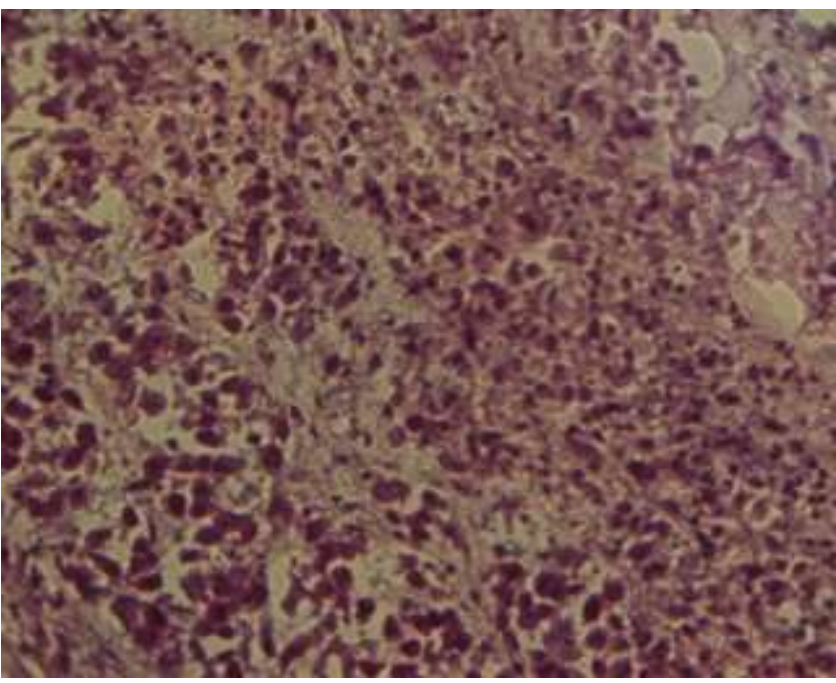

Figure 6: Original PSMA image.

TABLE III: ESTIMATION OF INITIAL AND FINAL PARAMETERS, AND ESTIMATION OF THRESHOLDS

\begin{tabular}{|l|l|}
\hline $\begin{array}{c}\text { Initial parameters } \\
\text { Estimated by K-mean }\end{array}$ & Estimated parameters by ML \\
$\mu_{1}^{0}=37$, & $\mu_{1}=31, \quad p_{1}=0.15$ \\
$p_{1}=0.22$ & $\mu_{2}=73, p_{2}=0.35$ \\
$\mu_{2}^{0}=63, p_{2}^{0}=0.54$ & $\mu_{3}=138 p_{3}=0.50$ \\
$\mu_{3}^{0}=132, p_{3}^{0}=0.23$ & \\
\hline & \\
& \\
&
\end{tabular}

Figure 7: Thresholded images.

\section{v. Conclusion}

Prostate cancer is the second most common cancer in men. In this paper we presented a PSMA image thresholding method based on the Gamma distribution. The maximum likelihood estimator is used in order to estimate the histogram parameters of the PSMA images. Thresholds between classes (modes) are then estimated by minimizing the discrimination error between the classes of pixels in PSMA image. The experimental results on PSMA prostate images using this technique showed good thresholding of images. As future work, we will use the threshold PSAM images in order to extract the feature of each region and then use pattern recognition techniques for object classification.

\section{Acknowledgment}

This work is supported by the National Plan for Service and Technology (NPST), King Saud University, Riyadh, Saudi Arabia, project BIO-1905.

\section{References}

[1] A. Sahirzeeshan, V. Robert, I.E. Jonathan, C. Christhunesa, M. Anant , Selective invocation of shape priors for deformable segmentation and morphologic classification of prostate cancer tissue microarrays, Computerized Medical Imaging and Graphics, Volume 41, April 2015, Pages 3-13.

[2] A. El-Zaart, D. Ziou, S. Wang, Q. Jiang (2002). 'Segmentation of SAR images', Pattern Recognition Journal, Vol.35, No.3, pp. 713-24

[3] S. Doyle, M. Hwang, K. Shah, A. Madabhushi, J. Tomasezweski, M. Feldman. Automated grading of prostate cancer using architectural and textural image features. In: International symposium on biomedical imaging (ISBI). 2007. p.1284-7.

[4] R. Farjam, H. Soltanian-Zadeh, K. Jafari-Khouzani, R. Zoroofi. An image analysis approach for automatic malignancy determination of prostate pathological images. Cytom B (Clin Cytom) 2007;72(B):227-40.

[5] R.J. Ferrari., R.M. angayyan, R.A. Borges, A.F. FRERE. (2004): 'Segmentation of the fibro-glandular disc in mammograms using Gaussian mixture modeling', Med. Biol. Eng. Comput., 42, pp. 37887

[6] D. Ziou, (1994): 'Optimal Thresholding for Image Segmentation', Proceeding of the Second African Conference On Research in Computer Science, Burkian-Faso. 3 pages.

[7] A. El-Zaart, D. Ziou, S. Wang, Q. Jiang (1998) 'Oil Spill Detection on SAR Images', Technical Report, No..211,Department of Computer Science, University of Sherbrooke, Sherbrooke, Canada,

[8] M. Mazonakis, J. Damilakis, H. Varveris, , P. Prassopoulos, and N. Gourtsoyiannis, Image segmentation in treatment planning for prostate cancer using the region growing technique he British Journal of Radiology, 74 (2001), 243-248 E 2001

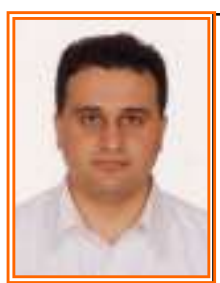

Ali El-Zaart was a senior software developer at Department of Research and Development, Semiconductor Insight, Ottawa, Canada during 20002001. From 2001 to 2004 , he was an assistant professor at the Department of Biomedical Technology, College of Applied Medical Sciences, King Saud University. From 2004-2011, he was an associate professor at the Department of Computer Science, College of computer and information Sciences, King Saud University. Presently, he is an associate professor at the Department of Mathematics and Computer Science, Faculty of science, Beirut Arab University. He has published numerous articles and proceedings in the areas of image processing, remote sensing, and 